\title{
Bayesian estimation in homodyne interferometry
}

\author{
Stefano Olivares \\ CNISM, UdR Milano Università, I-20133 Milano, Italy \\ Dipartimento di Fisica, Università di Milano, I-20133 Milano, Italy
}

\author{
Matteo G. A. Paris \\ Dipartimento di Fisica, Università di Milano, I-20133 Milano, Italy \\ CNISM, UdR Milano Università, I-20133 Milano, Italy \\ Institute for Scientific Interchange Foundation, I-10133 Torino, Italy
}

\begin{abstract}
We address phase-shift estimation by means of squeezed vacuum probe and homodyne detection. We analyze Bayesian estimator, which is known to asymptotically saturate the classical Cramér-Rao bound to the variance, and discuss convergence looking at the a posteriori distribution as the number of measurements increases. We also suggest two feasible adaptive methods, acting on the squeezing parameter and/or the homodyne local oscillator phase, which allow to optimize homodyne detection and approach the ultimate bound to precision imposed by the quantum Cramér-Rao theorem. The performances of our two-step methods are investigated by means of Monte Carlo simulated experiments with a small number of homodyne data, thus giving a quantitative meaning to the notion of asymptotic optimality.
\end{abstract}




\section{Introduction}

Quantum phase measurements cannot be described by means of a proper observable and different operational approaches have been introduced over the years 1, 2, 3, 4, 5, 6. On the other hand, from a practical point of view, phase detection of quantum fields is generally associated with interferometric devices, i.e., detection schemes aimed at the estimation of phase by measuring field- or intensity-based quantities with phasedependent statistics [7, 8, 9, 10, 11, 12, 13]. The art of interferomety, in turn, consists in answering to two question: a) How can the unknown phase be effectively retrieved from the data sample? and b) Which is the resulting precision? The first point amounts to the choice of an estimator, i.e., a function from the data sample to the set of possible values of the phase-shift. Among possible estimators Bayes [14] and maximum likelihood ones [15, 16 play a special role due to their asympotic (i.e., for large number of measurements) properties. The second point may be properly addressed in the framework of quantum estimation theory, which addresses the inference of a physical quantity which is not directly accessible by means of the measurement of a different observable, or a set of observables, somehow related to the quantity of interest. Quantum estimation is a powerful tool to infer a single parameter, as well to a set of parameters, up to the full reconstruction of the density matrix of an unknown quantum state, with or without the use of prior information [17, 18, 19]. Precision of any unbiased estimator is bounded by the inverse Fisher information of the probability distribution of the measurements outcomes, whereas the ultimate limit is written in term of the inverse Quantum Fisher Information (QFI).

In quantum optical systems, homodyne measurements of field quadratures and Gaussian signals play a leading role. Indeed, measurement of quadratures has been shown to achieve phase estimation for coherent states with precision bounded by the (classical) Fisher information [16]. This result have been further improved by looking for the optimal state achieving the ultimate bound related to the QFI [20]. Among the pure Gaussian states, squeezed vacuum has been found to be the most sensitive state at fixed energy and homodyne detection [21. Furthermore, it has been shown that the same signal allows optimal estimation of loss in bosonic channels 22 and of interaction parameters of single- and two-mode bilinear bosonic Hamiltonians [23]. Motivated by these results, in this paper we address optimal phase estimation by using Gaussian states, homodyne measurements and Bayesian estimation. We analyze the behavior for increasing number of measurements and show that optimality may be approached also with a limited number of runs upon using two-step methods acting on the squeezed vacuum probe and/or on the homodyne reference. Moreover, we prove that, in principle, the performances of double homodyne detection cannot beat the homodyne measurement ones, thus validating the conclusions of [21].

The paper is structured as follows. In Section 2 we briefly review local quantum estimation theory and the ultimate bounds to precision in the phase-shift estimation by Gaussian states. In Section 3 homodyne and double homodyne statistics are explicitly calculated for the phase shifted squeezed vacuum as input: this leads us to conclude that performances of the double homodyne detection cannot reach the limit imposed by QFI, while single homodyne does. Then, after describing our inference scheme, based on homodyne detection and Bayesian inference, the asymptotic limit for large number of collected data is studied in details, as well as the validity of the Gaussian approximation. Since the performances of this kind of inference protocol depend on the actual value of the (unknown) phase shift, we suggest two feasible two-step 
adaptive methods [24, 25], the first acting on the squeezing parameter, the other on the squeezing and local oscillator phases, that allow always to reach the optimal estimation. The results of simulated Monte Carlo experiments are reported in order to check convergence also for small data sample and give a quantitative meaning to the notion of asymptotic regime. Section 4 summarizes our results and draws some concluding remarks.

\section{Estimation of a phase shift}

Let us now consider a field mode undergoing a phase shift described by the unitary operator $U(\phi)=\exp (-i \phi G)$, with $G=a^{\dagger} a, a$ and $a^{\dagger}$ being the annihilation and creation field operators, respectively. Usually $\phi$ itself cannot be measured and a phase estimation problem appears. In order to infer the value of $\phi$ some phase-dependent observable $X$ is measured and an estimator for $\phi$, i.e., a function of the data sample $\{x\}$ is used. The aim of interferometry is to optimize the inference strategy by minimizing the uncertainty. In general, the lower bound to the variance $\operatorname{Var}[\phi]$ of any unbiased estimator is given by the Cramér-Rao theorem, which reads:

$$
\operatorname{Var}[\phi] \geq[F(\phi)]^{-1}
$$

where $F$ is the Fisher information:

$$
F(\phi)=\sum_{x} p(x \mid \phi)\left[\partial_{\phi} \log p(x \mid \phi)\right]^{2},
$$

$p(x \mid \phi)$ being the conditional probability of obtaining the outcome $x$ when the parameter has the value $\phi$. Since the conditional probabilities are given by $p(x \mid \phi)=$ $\operatorname{Tr}\left(\varrho_{\phi} E_{x}\right), \varrho_{\phi}=U(\phi) \varrho_{0} U^{\dagger}(\phi)$ being the quantum state of the system (actually depending on the initial preparation $\varrho_{0}$ ) and $E_{x}$ is the positive operator-valued measure (POVM) describing the measurement, Eq. (2) rewrites as:

$$
F(\phi)=\operatorname{Re} \sum_{x} \frac{\left[\operatorname{Tr}\left(\varrho_{\phi} E_{x} \Lambda_{\phi}\right)\right]^{2}}{\operatorname{Tr}\left(\varrho_{\phi} E_{x}\right)},
$$

where $\Lambda_{\phi}$ denotes the symmetric logarithmic derivative (SLD) operator:

$$
\partial_{\phi} \varrho_{\phi}=\frac{1}{2}\left(\Lambda_{\phi} \varrho_{\phi}+\varrho_{\phi} \Lambda_{\phi}\right) .
$$

Upon using Schwartz inequality in the Hilbert space one easily shows that the Fisher information in Eq. (3) is upper bounded by the so-called quantum Fisher information QFI $H(\phi)$ [20], i.e.:

$$
F(\phi) \leq H(\phi) \equiv \operatorname{Tr}\left(\varrho_{\phi} \Lambda_{\phi}^{2}\right) .
$$

The above equation, togehter with the Cramer-Rao theorem sets the ultimate, measurement-independent, bound to precision of any unbiased estimator involving quantum measurements.

In order to calculate the $\operatorname{SLD} \Lambda_{\phi}$, we first observe that if $\varrho_{0}$, and, in turn, $\varrho_{\phi}$ are pure states, then $\varrho_{\phi}=\varrho_{\phi}^{2}$ and $\partial_{\phi} \varrho_{\phi}^{2}=\left(\partial_{\phi} \varrho_{\phi}\right) \varrho_{\phi}+\varrho_{\phi}\left(\partial_{\phi} \varrho_{\phi}\right)$, thus, by comparison with Eq. (4), one finds $\Lambda_{\phi}=2 \partial_{\phi} \varrho_{\phi}$. More in general, we can expand $\varrho_{0}$ in its eigenvector 
basis $\left\{\left|\psi_{n}\right\rangle\right\}$, i.e., $\varrho_{0}=\sum_{n} p_{n}\left|\psi_{n}\right\rangle\left\langle\psi_{n}\right|$ (if $\varrho_{0}$ is a pure state, then $p_{n}$ reduces to a Kronecker delta), to write:

$$
\Lambda_{\phi}=\sum_{h k}\left\langle\psi_{h}\left|\Lambda_{\phi}\right| \psi_{k}\right\rangle\left|\psi_{h}\right\rangle\left\langle\psi_{k}\right|
$$

Then, since

$$
\partial_{\phi} \varrho_{\phi}=i \sum_{h k} G_{h k}\left(p_{h}-p_{k}\right)\left|\tilde{\psi}_{h}\right\rangle\left\langle\tilde{\psi}_{k}\right|
$$

with $G_{h k}=\left\langle\psi_{h}|G| \psi_{k}\right\rangle$, where $\left|\tilde{\psi}_{n}\right\rangle=U(\phi)\left|\psi_{n}\right\rangle$, we have:

$$
\frac{\Lambda_{\phi} \varrho_{\phi}+\varrho_{\phi} \Lambda_{\phi}}{2}=\frac{1}{2} \sum_{n} p_{n}\left(\Lambda_{\phi}\left|\tilde{\psi}_{n}\right\rangle\left\langle\tilde{\psi}_{n}|+| \tilde{\psi}_{n}\right\rangle\left\langle\tilde{\psi}_{n}\right| \Lambda_{\phi}\right)
$$

By taking the matrix elements of both sides in Eq. (4) we obtain:

$$
\left\langle\tilde{\psi}_{h}\left|\Lambda_{\phi}\right| \tilde{\psi}_{k}\right\rangle=\left\langle\psi_{h}\left|\Lambda_{0}\right| \psi_{k}\right\rangle=2 i G_{h k} \frac{p_{h}-p_{k}}{p_{h}+p_{k}},
$$

where $\Lambda_{\phi} \equiv U(\phi) \Lambda_{0} U^{\dagger}(\phi)$. As a consequence, $H(\phi)=\operatorname{Tr}\left(\varrho_{\phi} \Lambda_{\phi}^{2}\right)=\operatorname{Tr}\left(\varrho_{0} \Lambda_{0}^{2}\right)$, i.e., the QFI does not depend on the value of the unknown shift $\phi$. The explicit evaluation of the QFI $H=H(\phi)=H(0)$ leads to:

$$
H=4 \sum_{n s} p_{n} \frac{\left(p_{n}-p_{s}\right)^{2}}{\left(p_{n}+p_{s}\right)^{2}} G_{n s}^{2}
$$

where we used $G_{n s}=G_{s n}$. The maximum is obtained for the probe excited in a pure state. In this case, as described above, $\Lambda_{\phi}=2 \partial_{\phi} \varrho_{g}$ and, by substitution into Eq. (5), we obtain $H=4 \Delta G^{2}$, i.e., the QFI is proportional to the fluctuations of the Hamiltonian $G$ and the ultimate bound of $\operatorname{Var}[\phi]$ becomes:

$$
\operatorname{Var}[\phi]=\left(4 \Delta G^{2}\right)^{-1} \text {. }
$$

It is worth noticing that besides the number operator the above considerations hold for a general Hamiltonian generator $G[23]$.

Let us now come back to the problem of estimating $\phi$ by measurements on $\varrho_{\phi}$. Our aim is to effectively estimate the phase shift at fixed energy upon optimizing the measurement over detection strategies and probe states $\varrho_{0}$. Of course, the ultimate precision is bounded by the quantum Cramér-Rao relation (11), which depends on the probe state we employ. In turn, the first stage of the optimization procedure is to find the best probe, which maximizes the QFI at fixed energy. We focus our attention onto the set of pure states and, more precisely, on Gaussian pure states, whose generic element is a squeezed-displaced vacuum state given by:

$$
\varrho_{0}=D(\alpha) S(\xi)|0\rangle\langle 0| S^{\dagger}(\xi) D^{\dagger}(\alpha),
$$

$D(\alpha)=\exp \left(\alpha a^{\dagger}-\alpha^{*} a\right)$ and $S(\xi)=\exp \left(\frac{1}{2} \xi a^{\dagger}-\frac{1}{2} \xi^{*} a^{2}\right), \alpha, \xi \in \mathbb{C}$, being the displacement and squeezing operators, respectively. In order to maximize the QFI we look for the state maximizing the energy fluctuations at fixed probe energy $\operatorname{Tr}\left[\varrho_{0} a^{\dagger} a\right]=\sinh ^{2} r+|\alpha|^{2}$,

$$
\begin{aligned}
\Delta G^{2}= & \frac{1}{2} \sinh ^{2}(2 r)+e^{2 r}\{\operatorname{Re}[\alpha] \cos \varphi+\operatorname{Im}[\alpha] \sin \varphi\}^{2} \\
& -e^{-2 r}\{\operatorname{Re}[\alpha] \sin \varphi-\operatorname{Im}[\alpha] \cos \varphi\}^{2},
\end{aligned}
$$


where we put $\xi=r e^{-2 i \varphi}$. By using Lagrange multipliers one easily finds $|\alpha|=0$ : the maximum sensitivity is achieved when all the available energy is used to squeezed the vacuum. Then we have: $\Delta G^{2}=\frac{1}{2} \sinh ^{2}(2 r)$ and thus

$$
\operatorname{Var}_{\text {opt }}[\phi]=\left[2 \sinh ^{2}(2 r)\right]^{-1},
$$

which represents the ultimate bound on precision of phase-shift estimation posed by quantum mechanics (for Gaussian probes) 21. Notice that Eq. (14) does not depend on the argument $\varphi$ of the complex squeezing parameter $\xi$ : without lack of generality we will assume $\varphi=\pi / 2$. In the next Section we will show how it is possible to attain the ultimate precision by means of homodyne detection and Bayesian inference.

\section{Phase-shift estimation via homodyne detection and Bayesian inference}

We consider a general scheme (see Fig. 1) where the probe state $\varrho_{0}$ undergoes a phaseshift and then the quadrature $x_{\psi}$ is measured by homodyne detection on the outgoing state, $\varrho_{\phi}$.

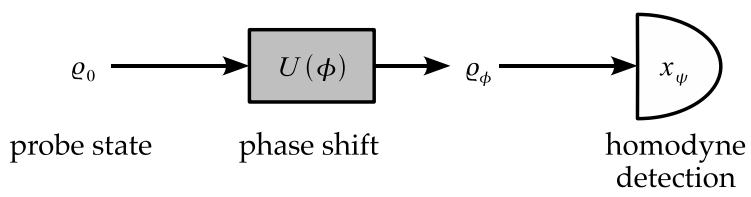

Figure 1. Scheme of phase estimation via homodyne detection: an input state $\varrho_{0}$ undergoes a phase shift $\phi$. The quadrature $x_{\psi}$ of the shifted state $\varrho_{\phi}=U(\phi) \varrho_{0} U^{\dagger}(\phi)$ is then measured by means of homodyne detection.

The aim of our scheme is to infer the actual value $\phi$ of the phase shift by processing the homodyne data. In order to evaluate the homodyne probability distribution we use the Wigner function formalism to describe our system. The Gaussian Wigner function associated with the state (12) is (we put $\alpha=0$ and $\varphi=\pi / 2$ ):

$$
W_{0}(\boldsymbol{X})=\frac{\exp \left[-\frac{1}{2} \boldsymbol{X}^{T} \boldsymbol{\sigma}_{0}^{-1} \boldsymbol{X}\right]}{2 \pi \sqrt{\operatorname{Det}\left[\boldsymbol{\sigma}_{0}\right]}},
$$

where $\boldsymbol{\sigma}_{0}=\frac{1}{4} \operatorname{Diag}\left(e^{-2 r}, e^{2 r}\right)$ is the $2 \times 2$ covariance matrix. After the phase shift (see Fig. 1), the state $\varrho_{\phi}$ is still described by a Gaussian Wigner function $W_{\phi}(\boldsymbol{X})$ of the form (15), but with covariance matrix $\boldsymbol{\sigma}_{\phi}$ given by:

$$
\begin{aligned}
& {\left[\boldsymbol{\sigma}_{\phi}\right]_{11}=\frac{1}{4}\left(e^{2 r} \cos ^{2} \phi+e^{-2 r} \sin ^{2} \phi\right),} \\
& {\left[\boldsymbol{\sigma}_{\phi}\right]_{22}=\frac{1}{4}\left(e^{-2 r} \cos ^{2} \phi+e^{2 r} \sin ^{2} \phi\right),} \\
& {\left[\boldsymbol{\sigma}_{\phi}\right]_{12}=\left[\boldsymbol{\sigma}_{\phi}\right]_{21}=\frac{1}{4} \sinh (2 r) \sin (2 \phi) .}
\end{aligned}
$$

At this point the quadrature $x_{\psi}=\frac{1}{2}\left(e^{-i \psi} a+e^{i \psi} a^{\dagger}\right)$ is measured by means of homodyne detection on repeated preparation of the probe state, thus obtaining a data sample $\{x\}$. Each outcome is distributed according to the homodyne probability distribution, 
which can be calculated starting from the Wigner function $W_{\phi}(\boldsymbol{X})$ as follows:

$$
p_{\phi}(x, \psi)=\int_{\mathbb{R}} d y W_{\phi}\left(R_{\psi} \boldsymbol{X}\right),
$$

where $R_{\psi}$ is a rotation matrix and $\boldsymbol{X}^{T}=(x, y)$. Since we put $\varphi=\pi / 2$, we choose to measure the quadrature with $\psi=0$. We have:

$$
p_{\mathrm{H}}(x \mid \phi) \equiv p_{\phi}(x, 0)=\frac{1}{\sqrt{2 \pi \Sigma_{\phi}^{2}}} \exp \left(-\frac{x^{2}}{2 \Sigma_{\phi}^{2}}\right)
$$

where:

$$
\Sigma_{\phi}^{2}=\frac{1}{4}\left[e^{-2 r} \cos ^{2} \phi+e^{2 r} \sin ^{2} \phi\right] .
$$

The Fisher information of the distribution (20) is given by:

$$
F_{\mathrm{H}}(\phi)=\int_{\mathbb{R}} d x p_{\mathrm{H}}(x \mid \phi)\left[\partial_{\phi} \log p_{\mathrm{H}}(x \mid \phi)\right]^{2}=\frac{\sinh ^{2}(2 r) \sin ^{2}(2 \phi)}{8\left(\Sigma_{\phi}^{2}\right)^{2}} .
$$

Remarkably, from Eq. (22) we have that the Fisher information of homodyne distribution may be equal to the QFI upon the choice of a suitable squeezing of the probe state:

$$
r=-\frac{1}{2} \log \tan \phi
$$

or, at fixed squeezing, for a specific value of the phase shift:

$$
\phi_{\mathrm{H}}=\frac{1}{2} \operatorname{arcos} \tanh 2 r .
$$

Correspondingly, the minumum variance $\operatorname{Var}_{\mathrm{H}}[\phi]$ achievable by a suitable processing of homodyne data mat saturate $\forall \phi$ to the ultimate bound (14).

Before going to the Bayesian inference from of homodyne data, we notice that if we use double-homodyne detection we have no improvement in phase-shift estimation. Double homodyne statistics is described by the coherent state POVM $\Pi_{z}=\pi^{-1}|z\rangle\langle z|$, $z \in \mathbb{C}$; the probability distribution is thus given by: $p_{\mathrm{D}}(z \mid \phi)=\pi^{-1}|\langle z|U(\phi) S(\xi)| 0\rangle|^{2}$ i.e.:

$$
p_{\mathrm{D}}(z \mid \phi)=\frac{\exp \left\{-|z|^{2}-\tanh r \operatorname{Re}\left[z^{2} e^{2 i \phi}\right]\right\}}{\pi \cosh r},
$$

where we already set $\xi=-r$. The corresponding Fisher information reads as follows:

$$
F_{\mathrm{D}}(\phi)=\int_{\mathbb{C}} d^{2} z p_{\mathrm{D}}(z \mid \phi)\left[\partial_{\phi} \log p_{\mathrm{D}}(z \mid \phi)\right]^{2}=4 \sinh ^{2} r
$$

that is $F_{\mathrm{D}}(\phi) \leq F_{\mathrm{H}}(\phi), \forall \phi$ : the use of double homodyne detection does not bring any improvement of the phase-shift measurement. This result agrees with the conclusions of [21], where the author considered double homodyne detection with squeezed vacuum as probe and an auxiliary squeezed state in the other input port.

We stress that $p(x \mid \phi)$ allows us to infer the probability of the homodyne outcome $x$ once the value of $\phi$ is assigned. In our case, the value of $\phi$ is just the quantity we want to estimate and, in turn, we are interested in the conditional a posteriori probability distribution $p_{\mathrm{M}}(\phi \mid\{x\})$ of $\phi$ given the the sample $\{x\}=\left\{x_{1}, \ldots, x_{M}\right\}$ of homodyne data. This can be obtained by means of Bayesian inference, as we will see in the following. 


\subsection{Bayesian inference}

If $x$ is the random variable associated with the outcome of the homodyne detection, then the Bayes' theorem states that:

$$
p(x \mid \phi) p(\phi)=p(\phi \mid x) p(x)
$$

where $p(\cdot \mid \cdot)$ are the conditional probabilities, $p(\phi)=2 / \pi$ is the prior assuming no a priori information, and $p(x)$ the overall probability to observe $x$. In turn, upon inverting Eq. (26) we obtain the conditional a posteriori probability $p(\phi \mid x)$ of $\phi$ given the outcome $x$. After $M$ independent homodyne measurement the a posteriori probability is given by

$$
p_{\mathrm{M}}(\phi \mid\{x\})=\frac{1}{\mathcal{N}} \prod_{k=1}^{M} p\left(x_{k} \mid \phi\right),
$$

$\mathcal{N}$ being the normalization factor:

$$
\mathcal{N}=\int_{0}^{\frac{\pi}{2}} d \phi p_{\mathrm{M}}(\phi \mid\{x\})
$$

If $M \gg 1$, then (27) rewrites as:

$$
p_{\mathrm{M}}(\phi \mid\{x\}) \stackrel{M \gg 1}{\simeq} \frac{1}{\mathcal{N}} \prod_{x} p(x \mid \phi)^{M p\left(x \mid \phi^{*}\right)} \equiv p(\phi \mid M)
$$

where $\phi^{*}$ stands for the actual (unknown) value of the phase shift. In order to write Eq. (29) we have used the law of large numbers and written the number of occurrences of the outcome $x$ as $M p\left(x \mid \phi^{*}\right)$. In this limit probability (29) can be explicitly calculated as follows:

$$
\begin{aligned}
p(\phi \mid M) & =\frac{1}{\mathcal{N}} \exp \left\{M \int d x p\left(x \mid \phi^{*}\right) \log p(x \mid \phi)\right\} \\
& =\frac{1}{\mathcal{N}} \frac{1}{\left(2 \pi \Sigma_{\phi}^{2}\right)^{M / 2}} \exp \left\{-\frac{M \Sigma_{\phi^{*}}^{2}}{2 \Sigma_{\phi}^{2}}\right\},
\end{aligned}
$$

where we used $\log \Pi_{x} \rightarrow \int d x$. We note that the quantity $S\left(\phi \mid \phi^{*}\right)=$ $-\sum_{x} p\left(x \mid \phi^{*}\right) \log p(x \mid \phi)$ in (30) may be regarded as the relative entropy between the two distributions [16. In Fig. 2 the a posteriori distribution $p(\phi \mid M)$ is plotted for different values of the involved parameters as a function of $\phi$. It is worth noting that because of the asymmetric form of the distribution, a suitable estimator for the actual value $\phi^{*}$ of the phase shift is given by the maximum of the distribution (its mode, Mode $[\phi])$ and not to its mean: $\bar{\phi}=\int_{0}^{\frac{\pi}{2}} d \phi \phi p(\phi \mid M)$. This can be easily seen by differentiating $p(\phi \mid M)$ with respect to $\phi$ :

$$
\partial_{\phi} p(\phi \mid M)=\frac{M p(\phi \mid M) F(\phi)}{8 \sin (2 \phi)}\left[\cos (2 \phi)-\cos \left(2 \phi^{*}\right)\right],
$$

i.e., $P(\phi \mid M)$ has a maximum at $\phi=\phi^{*}$. However, as $M$ increases the mode and the mean become the same and Eq. (31) can be approximated by a Gaussian distribution 

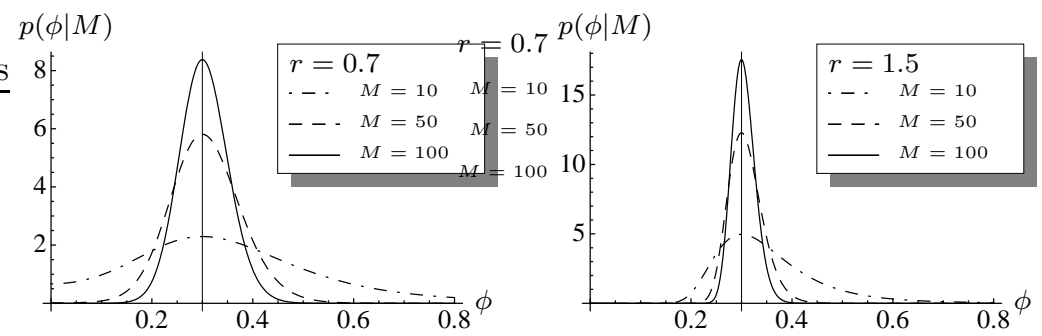

Figure 2. A posteriori distribution $p(\phi \mid M)$ for different values the number of data $M$ ad squeezing parameter $r$. The vertical line is the actual value of the phase shift $\phi^{*}=0.3$.
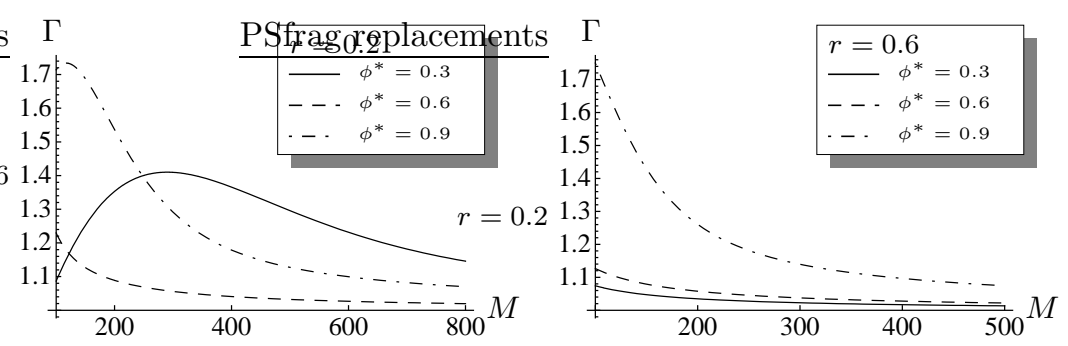

Figure 3. Plots of the ratio $\Gamma=\Sigma_{\mathrm{B}}^{2} / \Sigma_{g}^{2}$ for two values of the squeezing parameter $r$ and different $\phi^{*}$. The range of validity of Gaussian approximation strongly depends on the involved parameters. In particular, the less is the difference between $\phi^{*}$ and the optimal phase $\phi_{\mathrm{H}}$ given $r$ [see Eq. [24] ], the larger is the range of validity of this approximation (in the plots we have $r=0.2 \rightarrow \phi_{\mathrm{H}}=0.59$ and $r=0.6 \rightarrow \phi_{\mathrm{H}}=0.29$ ).

[26] with mean $\phi^{*}$ and variance $\Sigma_{g}^{2}$ given by:

$$
\begin{aligned}
\Sigma_{g}^{2} & =-\left[\left.\frac{1}{p\left(\phi^{*} \mid M\right)} \frac{d^{2} p(\phi \mid M)}{d \phi^{2}}\right|_{\phi=\phi^{*}}\right]^{-1} \\
& =\frac{1}{M}\left[\left.\sum_{x} \frac{1}{p\left(x \mid \phi^{*}\right)} \frac{d^{2} p(x \mid \phi)}{d \phi^{2}}\right|_{\phi=\phi^{*}}\right]^{-1}=\frac{1}{M F\left(\phi^{*}\right)},
\end{aligned}
$$

where we substituted Eq. (30) into Eq. (33) and $F\left(\phi^{*}\right)$ is the Fisher information of the probaility distribution $p\left(x \mid \phi^{*}\right)$. The factor $M^{-1}$ follows from taking the data sample as a collection of $M$ mutually independent measurements, which, indeed, leads to an ensemble average over $M$ different copies of the system. Finally, we notice that, as one may expect, the variance and, thus, the precision of the estimation depends on the true value $\phi^{*}$ itself.

Overall, the Bayes estimator is asymptotically unbiased and efficient, i.e., the variance $\operatorname{Var}[\phi]$ saturate the Cramér-Rao bound of Eq. (11): this is a consequence of the asymptotic normality of the a posteriori distribution (Laplace-Bernstein-von Mises theorem) [27, 28]. However, two questions arises. The first concerns the range of validity of the Gaussian approximation, which depends on both $\phi^{*}$ and the squeezing parameter $r$. This aspect is illustrated in Fig. 3 where we plot the ratio $\Gamma=\Sigma_{\mathrm{B}}^{2} / \Sigma_{g}^{2}, \Sigma_{\mathrm{B}}^{2}$ being the variance of the asymptotic distribution $p(\phi \mid M)$. For fixed $r$, one finds that 


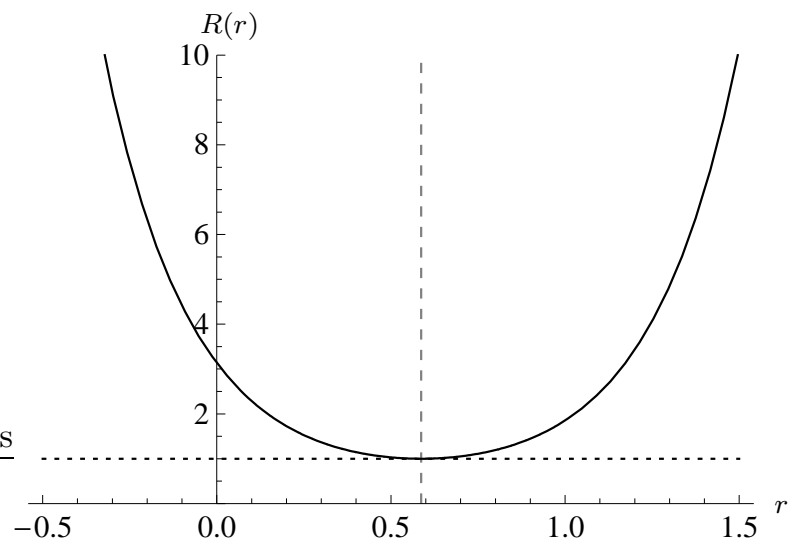

Figure 4. Plot of the ratio $R(r)=M \Sigma_{g}^{2}(r) / \operatorname{Var}_{\mathrm{opt}}\left[\phi^{*}\right]$ (see text) as a function of $r$ and for $\phi^{*}=0.3$. The vertical dashed line indicates $r_{\mathrm{opt}}$.

the less is the difference between $\phi^{*}$ and the optimal phase $\phi_{H}$ given $r$ [see Eq. (24)], the larger is the range of validity of this approximation. On this observation is also based the two-step adaptive method we will describe below. The second question is whether the Bayes estimator may saturate also the quantum Cramér-Rao bound, i.e., whether the Fisher information of $p(x \mid \phi)$ may be equal to the QFI, thus leading to phase-shift estimation with precision at the ultimate quantum limit. As what concerns this point we notice that, being the variance of Bayes estimator dependent on the true value of the phase shift, some kind of feedback should be unavoidably involved. In the following we will describe two possible adaptive mechanisms, acting on the squeezing parameter of the probe or on the homodyne local oscillator and squeezing phase, respectively.

\subsection{Examples of two-step methods to achieve ultimate precision}

Adaptive methods for Bayesian estimation allow to always attain the ultimate bound on precision and have been investigated in the case of large ensembles and qubit systems [29, 30. Here we propose two realistic and feasible setups exploiting the interferometric features of homodyne detection.

The first scheme is based on the fact that the variance $\Sigma_{g}^{2}(r)$ may achieve the optimal value $M^{-1} \operatorname{Var}_{\text {opt }}\left[\phi^{*}\right]$ of Eq. (14) employing a squeezed vacuum probe with parameter $r_{\mathrm{opt}}=-\frac{1}{2} \log \tan \phi^{*}$. Of course, setting $r=r_{\mathrm{opt}}$ requires the knowledge of the actual (unknown) value of the phase shift. However, one may obtain a rough estimate of $\phi^{*}$ upon building the distribution $p\left(\phi \mid M^{\prime}\right)$ with a fraction of the $M$, taking its maximum (Mode $[\phi]$ ) and then modify the probe state, tuning its squeezing to $r_{\text {opt }}$. In Fig. 4 we show the ratio $R(r)=M \Sigma_{g}^{2}(r) / \operatorname{Var}_{\text {opt }}\left[\phi^{*}\right]$ for the case $\phi^{*}=0.3$ : the smooth behavior of $R(r)$ ensures the convergence of the above mechanism. Tuning the squeezing parameter, however, could be a challenging task. On the other hand, also when $r$ and, thus, the energy are fixed, it is possible to achieve the optimal variance by tuning the squeezing phase $\varphi$ of the probe state or the phase $\psi$ of the homodyne quadrature. In fact, previously we set $\varphi=\pi / 2$ and $\psi=0$; if, on the contrary, we assign to these phases the generic values $\varphi$ and $\psi$, then we should simply apply the following change of variable in all the previous equations: $\phi \rightarrow \phi+\left(\varphi-\psi-\frac{\pi}{2}\right)$, that 

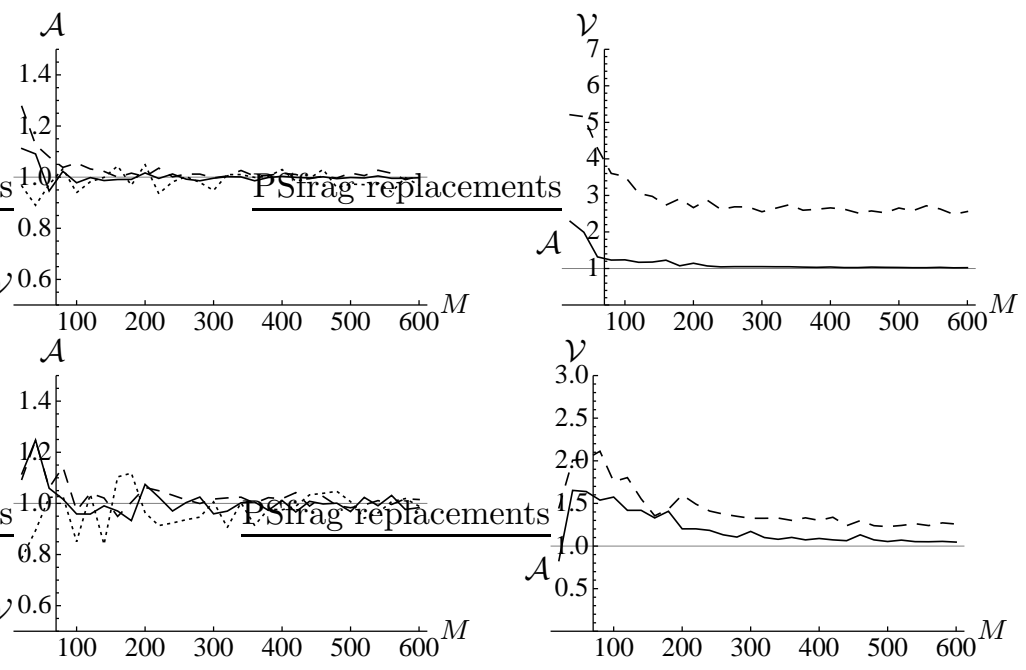

Figure 5. Bayesian estimation of the phase-shift from Monte Carlo simulated homodyne measurements: on the left the ratio $\mathcal{A}=\bar{\phi} / \phi^{*}$ and on the right $\mathcal{V}=\sqrt{\operatorname{Var}[\phi] / \operatorname{Var}_{\text {opt }}[\phi]}$ (right). The solid lines denote results obtained with the adaptive method acting on the homodyne local oscillator and squeezing phases; the dashed lines are obtained without the adaptive method. We set $r=0.6$ and $\phi^{*}=0.7$. In the case of the adaptive method, we used $\lfloor 3 \sqrt{M}\rfloor$ of the $M$ data to argue the phase-shift rough estimate (dotted line), then the left homodyne data are processed to assess $\bar{\phi}$ and $\operatorname{Var}[\phi]$. In both the experiments we use the same total number $M$ of data. Lower panel: the same as in the top panel for $r=0.3$.

is a translation of $\phi$ by the amount $\varphi-\psi-\frac{\pi}{2}$. Since the optimal angle $\phi_{\mathrm{H}}$ at fixed $r$ is given by Eq. (24), optimality is always achieved by choosing:

$$
\varphi-\psi=\phi_{\mathrm{H}}-\phi^{*}+\frac{\pi}{2} .
$$

As described above, we may obtain a rough estimate of $\phi^{*}$ by taking the maximum (Mode $[\phi])$ of $p(\phi \mid M)$, and, thus, we can tune the quantity $\varphi-\psi$ by means of adaptive control on the homodyne detection and/or the probe state, whose energy does not depends on $\varphi$.

In order to confirm convergence also for small data sample, we performed a set of Monte Carlo simulated experiments with the latter adaptive scheme. The results are shown in Fig. 5 for $r=0.6$ and $\phi^{*}=0.7$ (upper panel) and with reduced energy, $r=0.3$ (lower panel). In the experiment without adaptive method the whole sample of $M$ homodyne data, obtained as described in the first part of this section, is used to estimate $\bar{\phi}$ and $\operatorname{Var}[\phi]$ (dashed lines in Fig. 5 ). With the adaptive scheme (solid lines), $N_{\mathrm{r}}=\lfloor 3 \sqrt{M}\rfloor$ of the $M$ data sample are used to argue the phase-shift rough estimate, then the phase difference $\varphi-\psi$ is tuned according to Eq. (35) and the left homodyne data are processed to assess $\bar{\phi}$ and $\operatorname{Var}[\phi]$. Each point in Fig. [5 corresponds to the average over 20 repetitions. Of course, the effectiveness of the adaptive method depends on the value of the rough estimate: in this view, an increasing number of the outcomes devoted to the rough estimation, as the data sample becomes larger, allows the reduction of the $\operatorname{Var}[\phi]$ fluctuations, as one may verify, for example, by using a fixed value for $N_{\mathrm{r}}$. It is worth to note that in our simulations the rough estimate is obtained as Mode $[\phi]$, whereas the mean $\bar{\phi}$ is used for the final results: this is justified 
for the small $N_{\mathrm{r}}$ considered in the rough estimate and the larger number of the final estimation (the error introduced by this choice does not sensitively affect our results, as we verified also assessing the Pearson skewness coefficient $|\bar{\phi}-\operatorname{Mode}[\phi]| / \sqrt{\operatorname{Var}[\phi]})$.

\section{Conclusions}

In this paper we have shown how Bayesian inference techniques represent useful tools for phase estimation. Our analysis is based on homodyne detection with squeezed vacuum as a probe state, and Bayesian post-processing to infer the phase shift. In the asymptotic limit of a large number of measurements, our scheme saturates the Cramér-Rao bound to precision, i.e., the variance of the phase shift achieves the lower bound imposed by the inverse Fisher information. Moreover, we have shown that optimality may be approached also with a limited number of measurements by means of two-step methods acting on the squeezed vacuum probe and/or on the homodyne reference. These have been investigated by means of Monte Carlo simulated experiments, which show excellent results also in the case of small data samples. Our results, together with the recent advances in homodyne detection [31] lead us to conclude that the estimation protocol described in our paper may be suitable for experimental investigation, opening the way to information technology based on Gaussian states and phase encoding.

\section{Acknowledgments}

The authors thank A. Monras and M. G. Genoni for useful discussions. MGAP thanks Luca Pezze and Augusto Smerzi for useful discussions in the early stage of this work. This work has been partially supported by the CNR-CNISM convention. This article was completed at a time of drastic cuts to research budgets imposed by the Italian government; as a result research is becoming increasingly difficult in Italian universities and may in the near future be brought to a complete halt.

\section{References}

[1] D. T. Pegg and S. M. Barnett, Phys. Rev. A 39, 1665 (1989).

[2] J. W. Noh, A. Fougères and L. Mandel, Phys. Rev. Lett. 67, 1426 (1991).

[3] J. W. Noh, A. Fougères and L. Mandel, Phys. Rev. A 45, 424 (1992).

[4] J. W. Noh, A. Fougères and L. Mandel, Phys. Rev. A 46, 2840 (1992).

[5] G. M. D'Ariano, M. G. A. Paris, Phys. Rev. A , 48, R4039, (1993).

[6] G. M. D'Ariano, M. G. A. Paris, Phys. Rev. A 49, 3022, (1994).

[7] C. M. Caves, Phys. Rev. D 23, 1693 (1981)

[8] R. S. Bondurant and J. H. Shapiro, Phys. Rev. A 30, 2548 (1984).

[9] B. Yurke, S. L. McCall and J. R. Klauder, Phys. Rev. A 33, 4033 (1986)

[10] M. J. Holland and K Burnett, Phys. Rev. Lett. 71, 1355 (1993).

[11] V. Chickarmane and B. Bhawal, Phys. Lett. A 190, 22 (1994).

[12] M. G. A. Paris, Phys. Lett A 201, 132 (1995).

[13] G. M. D'Ariano, M. G. A. Paris, Phys. Rev. A 552267 (1997).

[14] L. Pezzè et al, Phys. Rev. Lett. 99, 223602 (2007).

[15] A. Lane et al Phys. Rev. A 47, 1667 (1993).

[16] Z. Hradil, et al., Phys. Rev. A 53, 3738 (1996).

[17] C. W. Helstrom, Quantum Estimation Theory (Academic Press, 1979).

[18] Quantum state estimation, Lect. Notes Phys. 649, M. G. A. Paris, J. Rehacek (Eds)

[19] Z. Hradil, Phys. Rev. A 51, 1870 (1995).

[20] S. L. Braunstein and C. M. Caves, Phys. Rev. Lett. 72, 3439 (1994).

[21] A. Monras, Phys. Rev. A 73, 033821 (2006). 
[22] A. Monras and M. G. A. Paris, Phys. Rev. Lett. 98, 160401 (2007).

[23] R. Gaiba, and M. G. A. Paris, arXiv:0802.1682

[24] D. W. Berry et al, Phys. Rev. A 63, 053804 (2001).

[25] S. Mancini et al, Phys. Rev. Lett. 80, 688 (1998).

[26] S. L. Braunstein, J. Phys. A: Math. Gen. 25, 3813 (1992).

[27] R. D. Gill, arXiv:0512443.

[28] L. Le Cam, Asymptotic Methods in Statistical Decision Theory (Springer-Verlag, 1986); H. L. Van Trees, Detection, Estimation and Modulation Theory - Part I (Wiley, 2001).

[29] R. D. Gill and S. Massar, Phys. Rev. A 61, 042312 (2000).

[30] M. Hayashi and K. Matsumoto in Asymptotic Theory of Quantum Statistical Inference: Selected Papers, Chapter 13, p. 162, M. Hayashi eds. (World Scientific, 2005), also available as quant-ph/0308150.

[31] V. Parigi et al., Science 317, 1890 (2007). 